\title{
Epidemiology of Measles in Bale Zone, Southeast Ethiopia: Analysis of Surveillance Data from 2013 to 2019
}

\author{
Geremew Tsegaye $\mathbb{D}^{\prime}$ \\ Yenealem Gezahagn ${ }^{2}$ \\ Shumi Bedada (D) ${ }^{3}$ \\ Naod Berhanu ${ }^{4}$ \\ Gemechu Gudina ${ }^{5}$ \\ Gadisa Mulatu ${ }^{3}$ \\ 'Ethiopian Field Epidemiology and \\ Laboratory Training Program, Faculty of \\ Public Health, Jimma University, Jimma, \\ Ethiopia; ${ }^{2}$ Department of Epidemiology, \\ Faculty of Public Health, Jimma \\ University, Jimma, Ethiopia; ${ }^{3}$ Disease \\ Control and Prevention Department, \\ Bale Zone Health Office, Robe, Ethiopia; \\ ${ }^{4}$ Field Epidemiology, Department of \\ Epidemiology, Faculty of Public Health, \\ Jimma, Ethiopia; ${ }^{5}$ Public Health \\ Emergency Management Department, \\ Oromia Regional Health Bureau, Addis \\ Ababa, Ethiopia
}

Background: Measles remains a cause of vaccine-preventable death in children worldwide. Reported cases in Ethiopiarepresent only a small proportion of expected cases, due to weak measles-based surveillance implementation. In this study, we aimed to analyze 7 years' measles-surveillance data from Bale zone, in order to indicate measles epidemiology and surveillance-related gaps.

Methods: This cross-sectional study was conducted from May to June 2019. The study population was all measles cases reported to the Bale Zone Health Office from 2013 to 2019. Data were abstracted from 7 years' measles line lists and case-based reports using a data-abstraction check-list. Data were analyzed using Microsoft Excel and Pivot software, and were presented in tables and graphs.

Results: Overall, 4,241 measles cases were reported from 2013 to 2019. Mean age was 7.15 years, and $50.6 \%$ were male. The most affected age-group was children $<4$ years of age. Analysis indicated that the case-fatality rate was $3.07 / 1,000$ population. Of the total cases reported, 248 (5.8\%) were IgM-confirmed. The highest prevalence of $141 / 100,000$ population was reported in 2019. Unvaccinated cases and those with unknown vaccination status numbered $890(21 \%)$ and 731 $(17.2 \%)$, respectively. The highest number of cases was reported from Ginir and Gololcha districts. Cases increased in autumn each year and peaked in May.

Conclusion: Measles is a major cause of morbidity and mortality in Bale, due to poor immunization coverage. Its case fatality is also high, excluding community deaths. Of all the districts included, Ginir reported the highest number of cases. Improving vaccination coverage, early preparedness for the annual epidemic cycle, and strengthening case-based surveillance are important interventions to reduce measles morbidity and mortality.

Keywords: Analysis, Bale zone, Ethiopia, Measles, Surveillance

\section{Introduction}

Measles is one of the most contagious diseases in humans, and is caused by a virus in the family Paramyxoviridae, genus Morbillivirus. Its incubation period is 10-14 days from exposure to symptom onset. Measles is characterized by fever, malaise, cough, coryza, and conjunctivitis, followed by a maculopapular rash., Complications are most common among children $<5$ years of age, and $30 \%$ of reported cases result in complications, such as pneumonia, diarrhea, encephalitis, and death. ${ }^{3}$

Despite complacency about measles vaccination in many developed parts of the world, ongoing transmission continues to pose real threats to unvaccinated and
Correspondence: Geremew Tsegaye Tel +25I-92-04I-0606

Email garamuts0606@gmail.com 
undervaccinated individuals globally. It also leads to individual and population health crises and heavy financial costs. ${ }^{4}$ Most measles-associated deaths occur in children aged 5 years and younger. Measles resulted in 90,000 and 110,000 deaths annually in 2016 and 2017, respectively. In high-income regions of the world, measles causes death one in 5,000 cases, but more than one per 100 cases in developing countries, which may reach $25 \%$ in high-risk areas. $^{4}$

In many African countries, measles remains a public health problem and among the top causes of death among those aged $<5$ years. In Africa, it is the leading cause of blindness in children, due to low immunization coverage and other prevailing living conditions. ${ }^{3}$ In 2013, about 171,178 cases were reported in the Africa region of the World Health Organization (WHO), ${ }^{5,6}$ with high rates among children $<1$ year of age. ${ }^{7}$

Measles is a leading cause of vaccine-preventable death and endemic in low vaccine-coverage countries. It follows a seasonal pattern, with increasing incidence in the dry season, ie, from November to May, ${ }^{6}$ and peaks during December to February. ${ }^{8}$

Measles continues to be one of the major causes of death and sickness of children in Ethiopia, and outbreaks are reported annually from different parts of the country. ${ }^{3,8}$ Ethiopia is committed to achieving the elimination of measles. Current incidence in Ethiopia is 50 cases/1,000,000 population per year, which is above the national target set in 2017 of $<1 / 1,000,000$ population per year. ${ }^{9}$ The case-fatality rate (CFR) is $3 \%-6 \%$, and in certain high-risk populations, CFRs reaching as high as $30 \%$ have been reported in infants aged $<1$ year of age. ${ }^{3}$ According to surveillance-data analysis conducted in Oromia regional state in 2012-2016, 26,908 cases and 288 deaths were reported. ${ }^{10}$

Risk factors of measles are inadequate vaccination, malnourishment, children with vitamin A deficiency and immunodeficiency due to HIV/AIDS, leukemia, alkylating agents, or corticosteroid therapy, regardless of immunization status, traveling to areas where measles is endemic or contact with travelers to endemic areas all carry a high risk of infectons. ${ }^{7}$

As a result of the existence of a highly effective measles vaccine, ${ }^{11} 4$ million measles-related deaths were averted in 2012-2014. ${ }^{9}$ The vaccine is live and attenuated, and two doses are recommended by the WHO to provide protection. $^{12}$ According to WHO Africa in 2015, for measles elimination in Africa, Ethiopia was among eleven countries with major challenges, large populations, insecurity, high incidence, frequent outbreaks, and leadership gaps in controlling the disease. ${ }^{12}$

According to the WHO, Ethiopia is among the six countries with the most unvaccinated infants -1.1 million in $2017 .{ }^{13}$ Ethiopia is among the nations with low measles-containing vaccine (MCV1) coverage, and MCV2 was given as supplementary immunization until recently. To get extra immunity, the government introduced a second vaccination (MCV2) into the routine immunization program for children in their second year of life in February 2019. Measles antibodies develop in approximately in 95\% of children vaccinated at 9-12 months of age and $98 \%$ of children vaccinated at 15 months of age. ${ }^{3,14}$

According to the WHO, measles surveillance is a key element to achieving elimination. ${ }^{15}$ The objectives of surveillance are to detect, identify, investigate, and manage outbreaks by identifying geographic areas and age-groups at high risk and evaluate vaccination strategies in order to improve measles control. ${ }^{7}$ In Ethiopia measles, case-based surveillance is part of the national Public Health Emergency Management (PHEM) system.

Case-based measles surveillance was initiated in Ethiopia in 2003. Depite being reported on weekly, the number of reported cases represents only a small proportion of expected cases. Measles cases usually come to health facilities often after they develop a complication. As a result, they are diagnosed with the complication rather than measles, which is one of the reasons for underreporting. ${ }^{8}$

Robust measles surveillance and periodic analysis of aggregated data is crucial in implementing public health prevention measures and to develop routine and supplementary immunization activities. The aim of this study was to analyze 7 years' (2013-2019) measles surveillance data of Bale zone by person, place, and time. The analysis includes vaccination status, laboratory confirmation, and CFRs.

\section{Methods}

\section{Study Area}

Bale is among 21 administrative zones of Oromia regional state. It is located in the southeastern part of Oromia and is situated between $5^{\circ} 11^{\prime} 03^{\prime \prime} \mathrm{N}$ to $8^{\circ} 09^{\prime} 27^{\prime \prime} \mathrm{N}$ and $38^{\circ} 12$ '04'E to $42^{\circ} 12^{\prime} 47^{\prime \prime} \mathrm{E}$ longitude, with an altitude that ranges from 300 to $4,377 \mathrm{~m}$ above sea level. Robe, the capital of the zone is situated $430 \mathrm{~km}$ from Addis Ababa. Bale has 18 districts and two city administrations. About 
383 health posts, 83 health centers, and four hospitals are found in the zone. The total population projected for 2019 was $1,963,416$.

\section{Study Design and Period}

This cross-sectional study was conducted from May 25 to June 25,2019 to analyze measles-surveillance reports of 2013-2019.

\section{Source and Study Populations}

All people in Bale during the study period were the source population, while all confirmed, epidemiologically linked, and clinically compatible measles cases reported during 2013-2019 were the study population.

\section{Inclusion and Exclusion Criteria}

Complete line lists and case-based reports of measles recorded in the database of the Bale PHEM department from 2013 to 2019 were included.

Line lists and case-based reports registered prior to January 2013 and after December 2019 and line lists with incomplete data were excluded.

\section{Sample Size and Sampling Procedure}

All 4,241 measles cases reporting to the Bale PHEM department from 2013 to 2019 were taken.

\section{Data-Collection Procedure and Analysis}

Secondary data on measles for 2013-2019 were abstracted using a format developed for this purpose. Data were collected from line lists and case-based and weekly integrated disease-surveillance and response reports. Data were abstracted in terms of person, place, time, case classification, vaccination status, and final outcome. The abstracted data were entered and analyzed using Microsoft Excel and Pivot software. ArcGIS version 10.1 was used to locate the area and derive spot maps of cases. Medians, standard deviations, and percentages were used to analyze surveillance data for 2013-2019. Prevalence, case fatality (CF), and vaccination status were analyzed. The data were described by person, place, and time. The results were finally presented in tables, graphs, and figures.

\section{Operational Definitions}

\section{Suspected Measles Case}

Any person with fever and maculopapular generalized rash and cough, coryza, or conjunctivitis OR any person in whom a clinician suspects measles.

\section{Confirmed Measles Case}

A laboratory-confirmed (positive for IgM antibodies) case or one epidemiologically linked to confirmed cases.

\section{Epidemiologically Linked Case}

A suspected case living in the same or in an adjacent district with a laboratory-confirmed case where there was a likelihood of transmission, with onset of rash of the two cases being within 30 days of each other.

\section{Death}

Any death from an illness in a confirmed case or epidemiologically linked cases within 1 month of the onset of rash.

\section{Discarded}

A suspected case that had been completely investigated, including the collection of adequate blood specimens $(5 \mathrm{~mL})$, but lacked serological evidence of recent infection (ie, IgM-negative).

\section{Clinical/Compatible}

A suspected case that had not had a blood specimen taken for serological confirmation, and could not be epidemiologically linked to a laboratory-confirmed case.

\section{Ethics}

An official letter was written by Jimma University School of Public Health and presented to Bale Zonal Health Office. A permission letter was obtained from the Bale PHEM department to access 7 years' zonal measles data to carry out this analysis. The privacy of the data was maintained by using it only for our study purposes.

\section{Results}

\section{Case Distribution by Age and Sex}

A total of 4,241 measles cases were reported by 475 health facilities and 20 districts to the Bale Zone Health Office during 2013-2019. The overall crude attack rate was 34/ 100,000 population. Mean age was $7.15 \pm 8.95$ years. Those aged $1-4$ years comprised $40 \%$, followed by those aged $5-$ 15 years, at $30 \%$ (Table 1 ).

Of all reported cases, 2,147 (50.6\%) were male and 2,094 (49.4\%) female, for a female:male ratio of 1:1.04. Sexspecific prevalence was calculated, and higher prevalence was seen among male cases $(2.27$ per 1,000$)$. Female prevalence was 2.2 per 1,000 . The trend showed almost equal distribution of cases between the sexes, with slight differences in 2013, 2015, and 2019, as shown in Figure 1. 
Table I Measles cases reported

\begin{tabular}{|c|c|c|c|c|c|c|c|c|}
\hline & \multirow{2}{*}{ Population } & \multirow[t]{2}{*}{ Total cases } & \multirow[t]{2}{*}{ Deaths } & \multirow[t]{2}{*}{$A R / 100,000$} & \multirow[t]{2}{*}{ CFR/I,000 } & \multirow[t]{2}{*}{ Age } & \multicolumn{2}{|c|}{ Total } \\
\hline & & & & & & & Cases, $\mathrm{n}$ & $\%$ \\
\hline 2013 & I,658,497 & 198 & 0 & 12 & 0 & & & \\
\hline 2014 & $1,703,762$ & 233 & 2 & 14 & 8.6 & $<$ I year & 503 & 12 \\
\hline 2015 & I,749,027 & 371 & 1 & 21.2 & 2.7 & I-4 years & 1,682 & 40 \\
\hline 2016 & I,794,940 & 259 & 1 & 14.4 & 3.9 & $5-15$ years & 1,260 & 30 \\
\hline 2017 & $\mathrm{I}, 840,746$ & 133 & 0 & 7.2 & 0 & $16-35$ years & 649 & 15.3 \\
\hline 2018 & $1,911,685$ & 275 & 0 & 14.4 & 0 & $36-49$ years & 126 & 3 \\
\hline 2019 & $1,963,4 \mid 6$ & 2,772 & 9 & 141.2 & 3.2 & $>50$ years & 21 & 0.5 \\
\hline Total & $12,622,073$ & $4,24 I$ & 13 & 33.6 & 3.1 & & $4,24 I$ & \\
\hline
\end{tabular}

Abbreviations: AR, attack rate; CFR, case-fatality rate.

\section{Age-Specific Prevalence by Time}

Prevalence was high in children aged $<1$ year, with highest prevalence of 30/1,000 reported in 2019. The secondmost affected age-group was 1-4 years, with highest prevalence of 4.29/1,000 in 2019. Prevalence was lower among those aged 15 years and above, with highest prevalence of 0.73/1,000 observed in 2019 (Table 2).

\section{Prevalence by District}

Of the 4,241 measles cases reported, the highest prevalence was reported in Ginir district $(77 / 10,000)$, followed by Dinsho $(45.4 / 10,000)$ and Golocha $(38.4 / 10,000)$. The lowest prevalence was reported in Gura Damole and
Gasera: $0.6 / 10,000$ and $0.9 / 10,000$, respectively, as shown in Figure 2.

The highest prevalence $(>50 / 10,000)$ was reported from Gindhir and Gololcha districts. The lowest prevalence $(<5 / 10,000)$ was reported from Rayitu and Guradamole districts. A spot map of the distribution of cases with prevalence per 10,000 is shown in Figure 3.

\section{Geographic Distribution}

The highest number of cases was reported from rural areas of the zone $(3,642,85.8 \%)$ cases, while only 599 (14.1\%) were reported from urban areas.

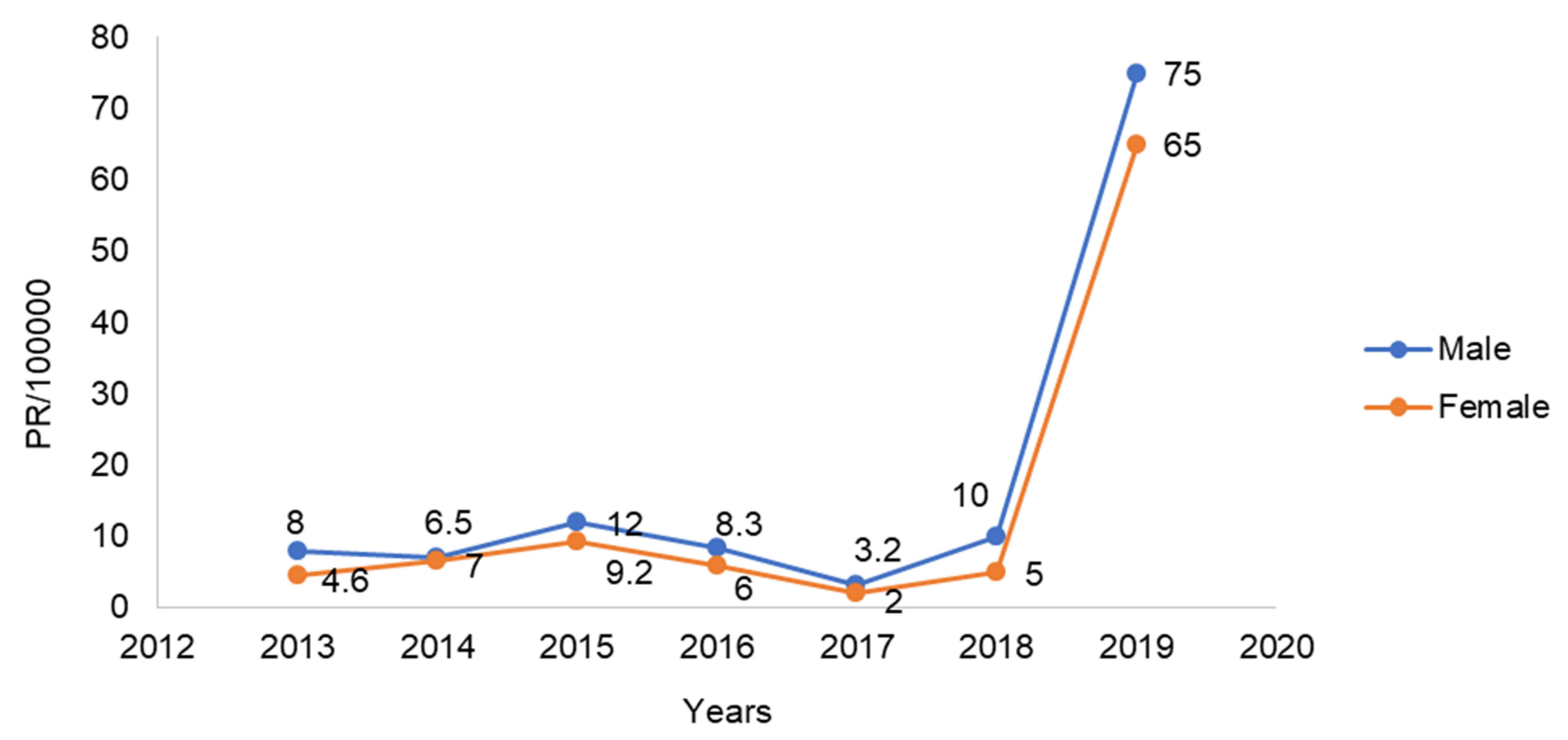

Figure I Measles trends by sex. Abbreviation: PR, prevalence. 
Table 2 Age-specific measles prevalence

\begin{tabular}{|l|c|c|c|c|c|c|}
\hline \multirow{2}{*}{} & \multicolumn{5}{|c|}{ Prevalence per I,000 population by age (years) } \\
\cline { 2 - 7 } & $<1$ & $\mathbf{I - 4}$ & $\mathbf{5 - 1 5}$ & $\mathbf{1 6 - 3 5}$ & $\mathbf{3 6 - 4 9}$ & $<\mathbf{5 0}$ \\
\hline 2013 & 1.17 & 0.31 & 0.09 & 0.08 & 0.03 & 0.02 \\
\hline 2014 & 2.96 & 0.31 & 0.09 & 0.13 & 0.00 & 0.00 \\
\hline 2015 & 5.31 & 0.42 & 0.22 & 0.09 & 0.03 & 0.03 \\
\hline 2016 & 6.36 & 0.35 & 0.12 & 0.02 & 0.02 & 0.02 \\
\hline 2017 & 3.47 & 0.21 & 0.06 & 0.01 & 0.00 & 0.00 \\
\hline 2018 & 2.34 & 0.33 & 0.13 & 0.10 & 0.01 & 0.01 \\
\hline 2019 & 29.80 & 4.29 & 1.32 & 0.73 & 0.01 & 0.01 \\
\hline Total & 51.41 & 6.21 & 2.04 & 1.16 & 0.10 & 0.10 \\
\hline
\end{tabular}

\section{Trends by Month}

The highest numbers of cases were reported in June $(1,344,31.6 \%)$, May (934, 22\%), and April (545, 12\%). Cases peaked in May, with fewer reported in October, November, and September, as shown in Figure 4.

\section{Trends in Prevalence by Time}

The highest numbers of suspected cases were seen in 2019 and 2015: 141 and 21 per 100,000, respectively. A remarkable decrement in suspected cases was reported in the year 2017: 7.2/100,000 In 2013, it was 11.4/ 100,000 (Figure 5).

\section{Final Case Classifications}

Of the total 4,241 cases reported during the study period,only 248 (5.8\%) were laboratory-confirmed for IgM, and $3,060(72.1 \%)$ were epidemiologically linked to confirmed cases. About 497 (11.3\%) were reported as being clinically compatible with measles, as shown in Figure 6.

\section{Laboratory Results}

A total of 993 serum samples were sent to Ethiopian Public Health Institute's central laboratory for confirmation: $248(25 \%)$ tested were positive for IgM, and 375 (37.8\%) were discarded (Figure 7).

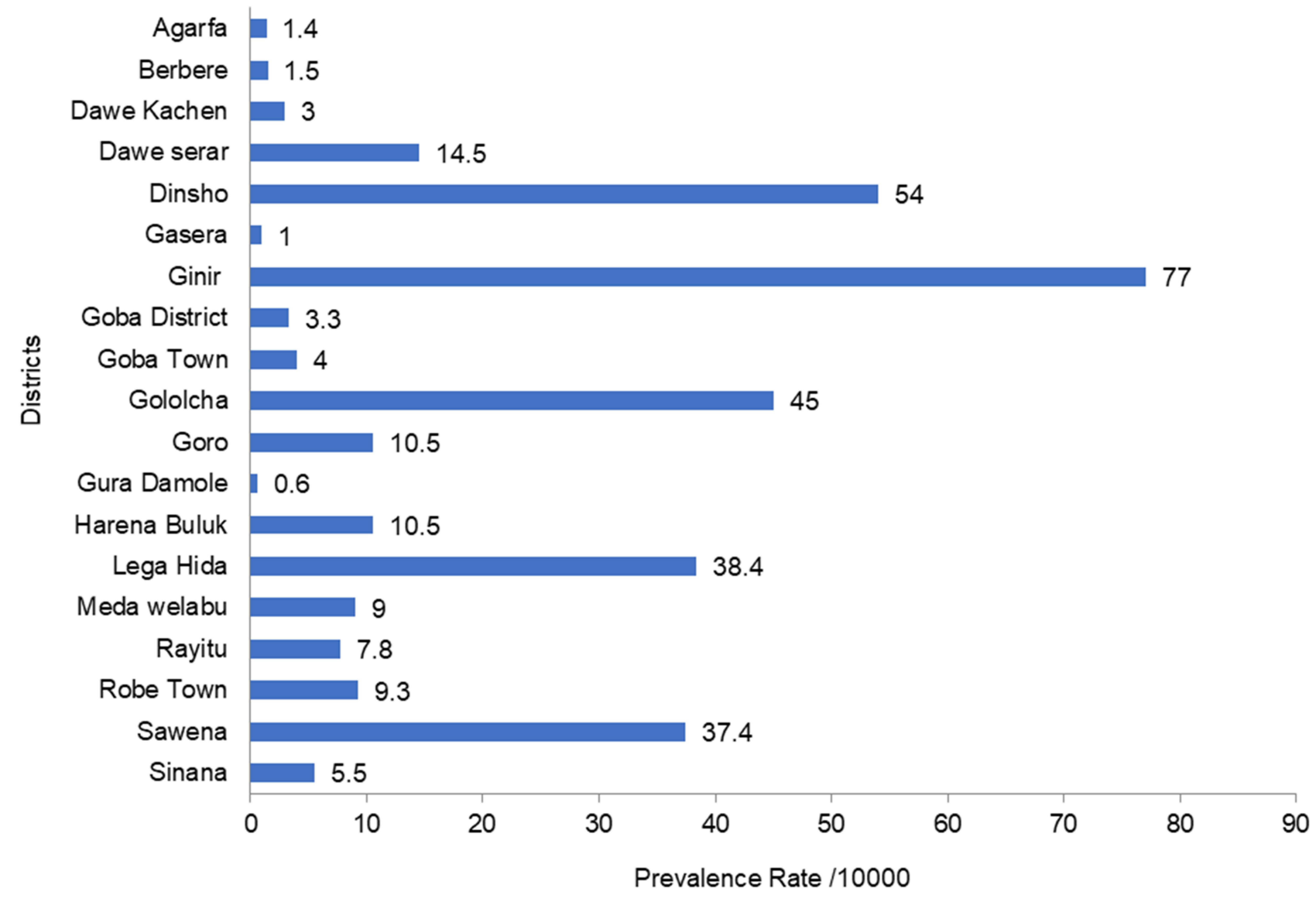

Figure 2 Measles distribution by prevalence per 10,000 population. 


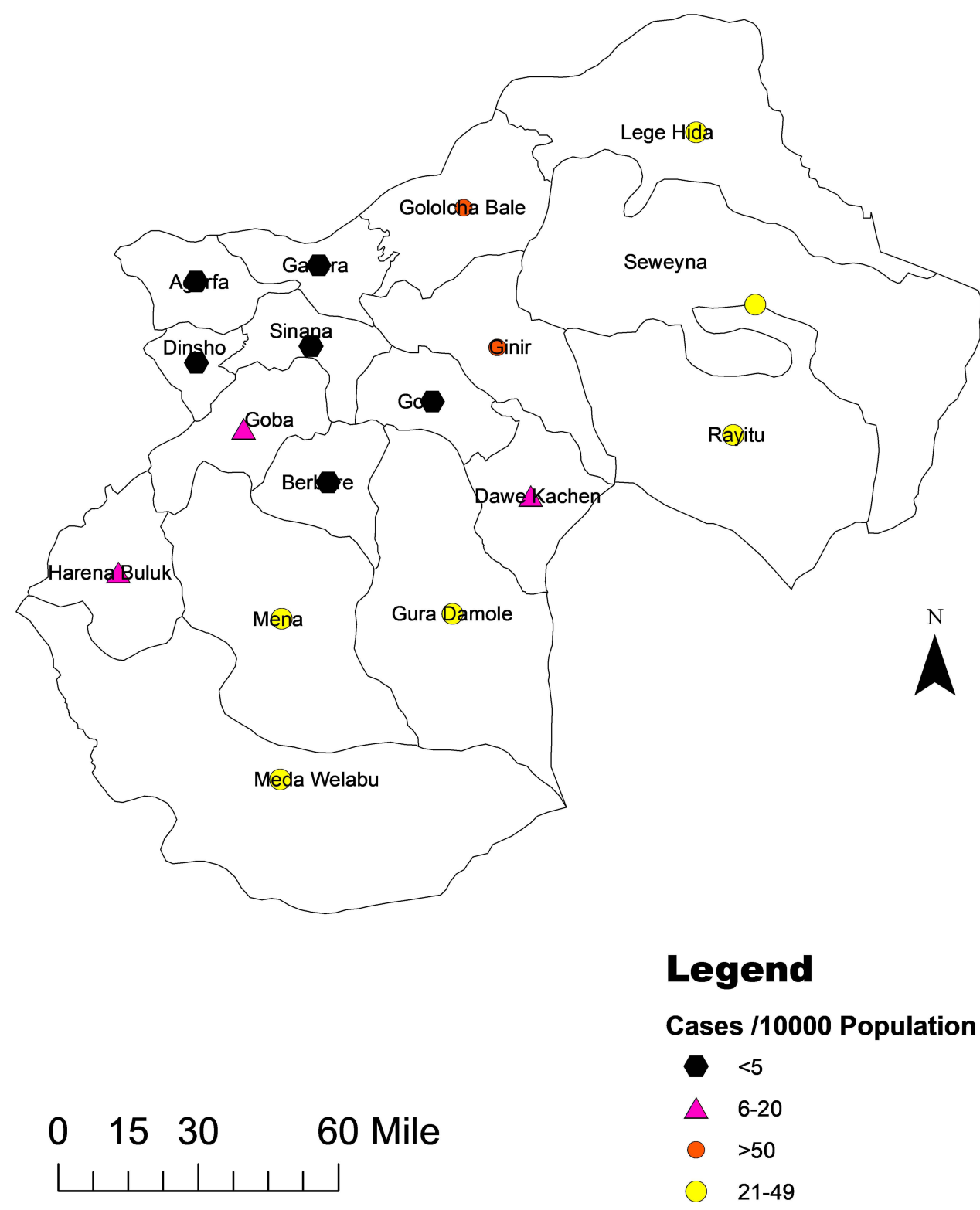

Figure 3 Measles distribution by prevalence per 10,000 population in Bale districts.

\section{Inpatient and Outpatient Cases}

In sum, $582(13.2 \%)$ cases were admitted as inpatients and $3,659(86.2 \%)$ suspected cases were treated as outpatients. The overall admission rate for treated cases was $16 \%$.

\section{Case-Fatality Rate}

Of all cases, the crude CFR was 3.07/1,000, and most (nine, 69.2\%) measles-related deaths were reported from Ginir district.

\section{Case Fatality by Sex and Age}

Fatality was higher among those of female sex than male: crude CFR 3.3/1,000 and 2.8/1,000, respectively. The highest age-specific fatality rates (four per 1,000) were observed among those aged $<1$ year and 5-15 years(Figure 8).

\section{Trends in Case Fatality by Year}

The greatest CFR occurred during 2014 (8.6/1,000), and in 2016 this was four per 1,000 . Measles-related deaths were not reported in 2013, 2017, or 2018 (Figure 9).

\section{Vaccination Status}

All in all, 2,619 (62\%) were vaccinated, of which 1,480 (34.4\%), 1,032 (24.3\%), and 108 (2.5\%) had received one, two, and three doses of MCV, respectively. There were $890(21 \%)$ patients with no vaccination history and 732 $(17.3 \%)$ with unknown vaccination status (Table 3 ). 


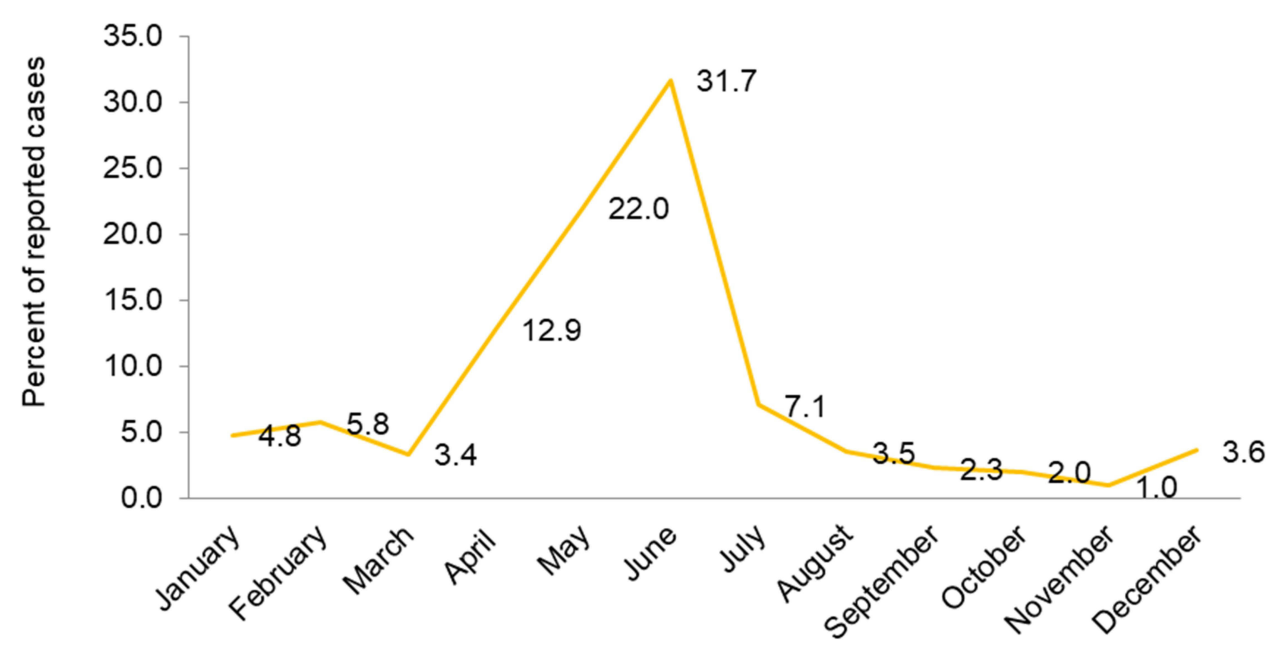

Months of the year

Figure 4 Cyclic patterns of measles cases.

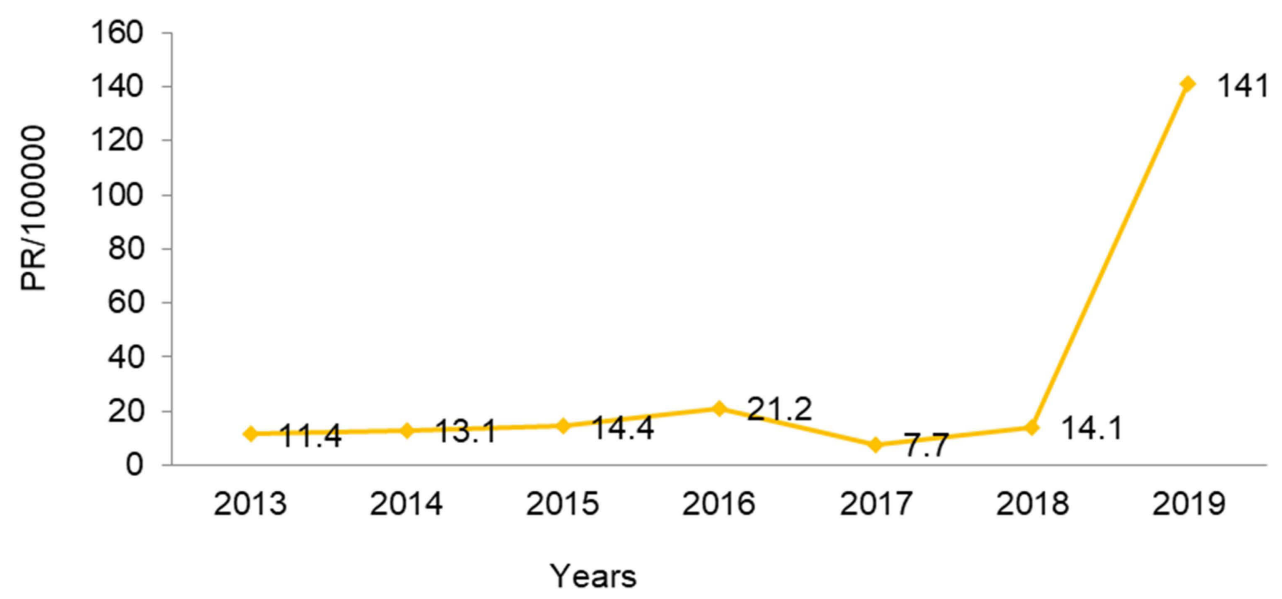

Figure 5 Trend of measles prevalence by year.

\section{Vaccination Status by Place}

Regarding unknown vaccination status, 390 (53.4\%) were from Ginir and 107 (14.6\%) from Gololcha. Vaccinated cases were also highest in Ginir, Gololcha, and Sewena: 981 (37.4\%), 400 (15.3\%), and 213 (8.1\%), respectively. Unvaccinated cases numbered $443(50 \%)$ in Ginir, 88 (10\%) in Dawe Serer, and 82 (9.2\%) in Lega Hida. Ginir woreda reported the highest number of both vaccinated and unvaccinated cases.

\section{Vaccination Status by Time}

Of the 890 unvaccinated cases, the highest proportions were reported in 2019 and 2014: 73.3\% and 9\%, respectively. Of those who had been vaccinated, the highest proportions (one or two) were reported in 2019 and
2015: $67.9 \%$ and $68 \%$, and $7.2 \%$ and $14 \%$, respectively. Lower proportions were reported in 2014: 2\% single doses and $1.5 \%$ of double doses. Overall, $41 \%$ of those with unknown vaccination status were reported in 2019, which is relatively high (Figure 10).

\section{Data Type}

Of the 4,241 suspected cases, 903 (21\%) were reported as case-based and 3,312 (64.9\%) as line lists.

\section{Discussion}

Surveillance-data analysis was performed to identify associations between reported health outcomes and patient or population characteristics to understand the local epidemiology and determine effective prevention or control measures. 


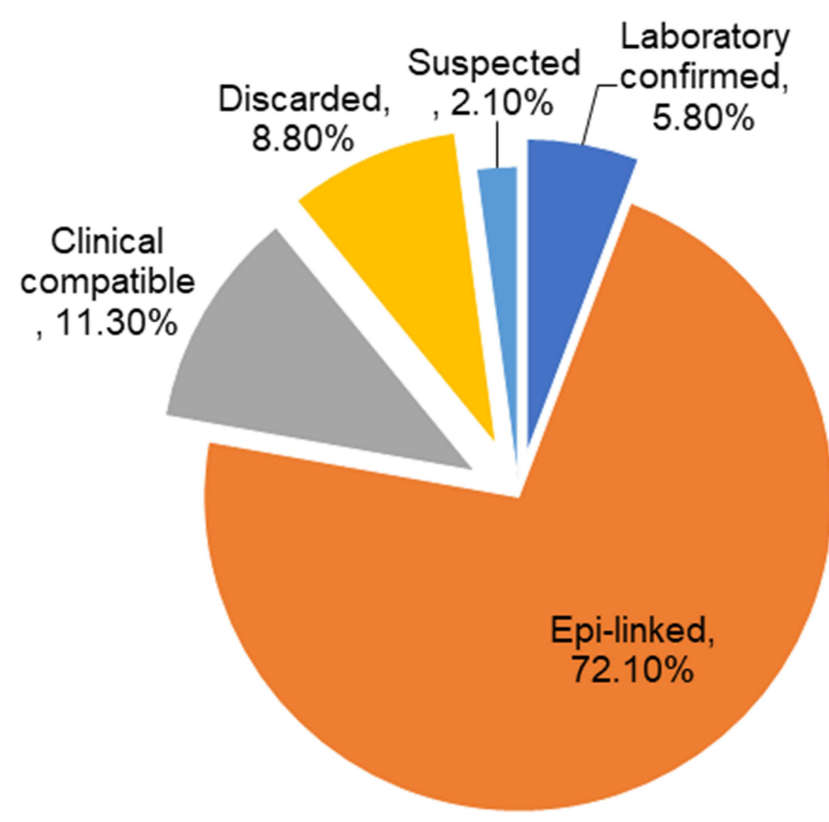

Figure 6 Final case classification of measles cases.
Periodic surveillance-data analysis is crucial to understanding significant reductions in incidence and mortality and to identify gaps in the surveillance system for possible correction.

The current analysis indicated that the most cases were observed among those aged $<1$ year, and cases were reported for all age categories. This finding is in line with a study conducted in Guji zone, which showed all age-groups - even $>15$ years - were affected ${ }^{16}$ and those aged 1-4 years most affected. ${ }^{17}$ This is because of malnutrition and inadequate vaccination status.

The highest measles prevalence was in 2015 and 2019: 21 and 141 per 100,000 , respectively. This is higher than studies conducted in Nigeria $(19 / 100,000)^{16}$ and Ethiopia (more than per 100,000/year over five years. ${ }^{18}$ This difference is possibly due to many susceptible persons and outbreaks occurring frequently in the zone. In addition, more than half the population of the zone are pastoralists,

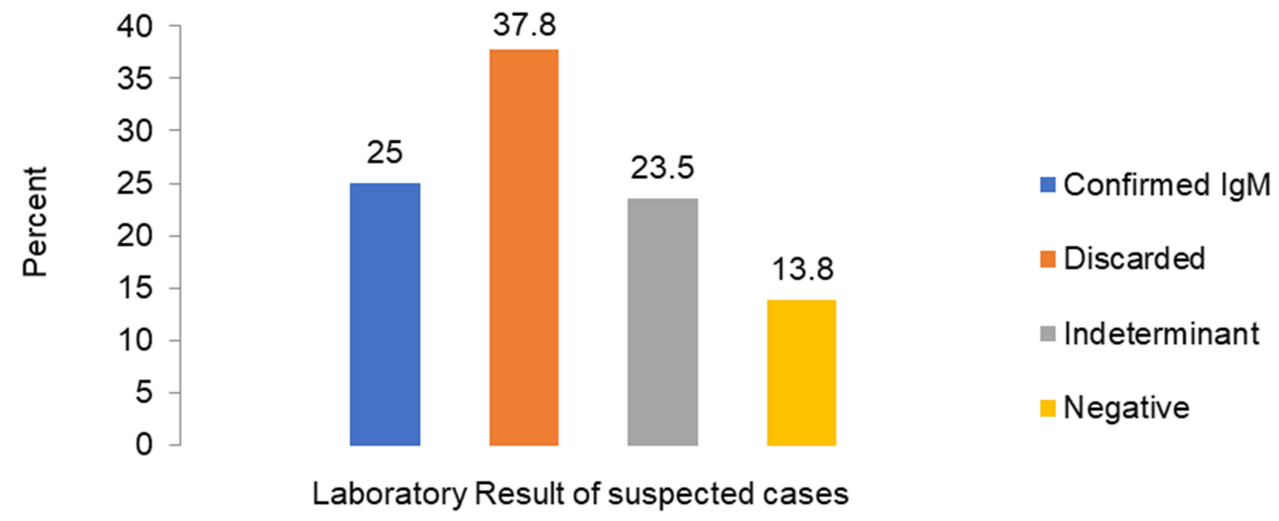

Figure 7 Percentage of samples tested and laboratory results.

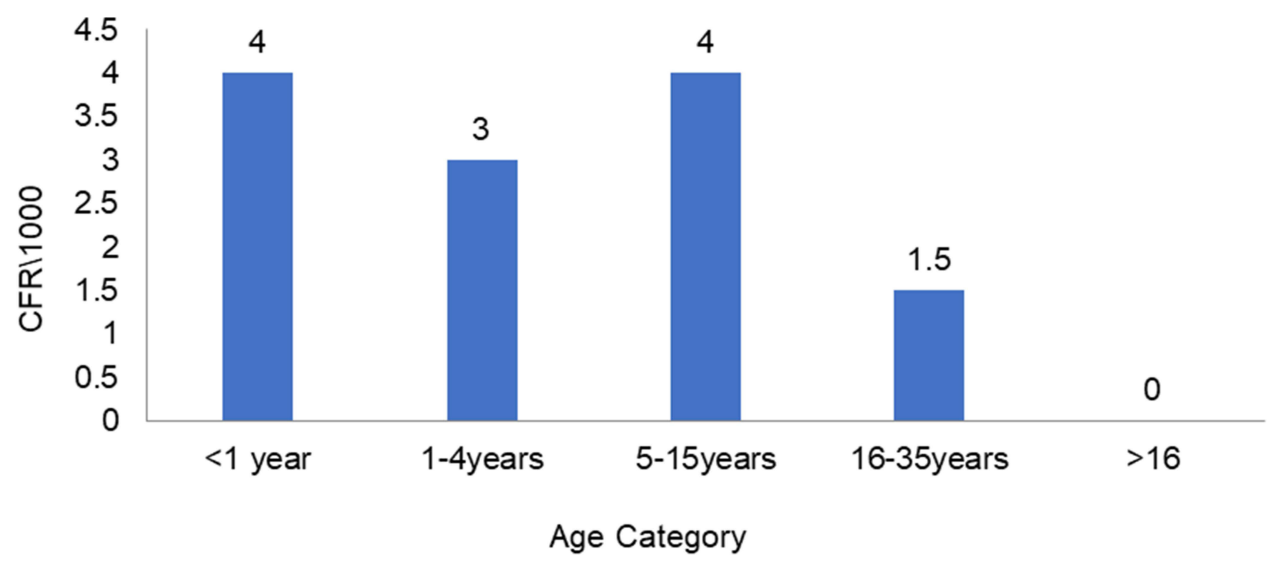

Figure 8 Case-fatality rate by age. 


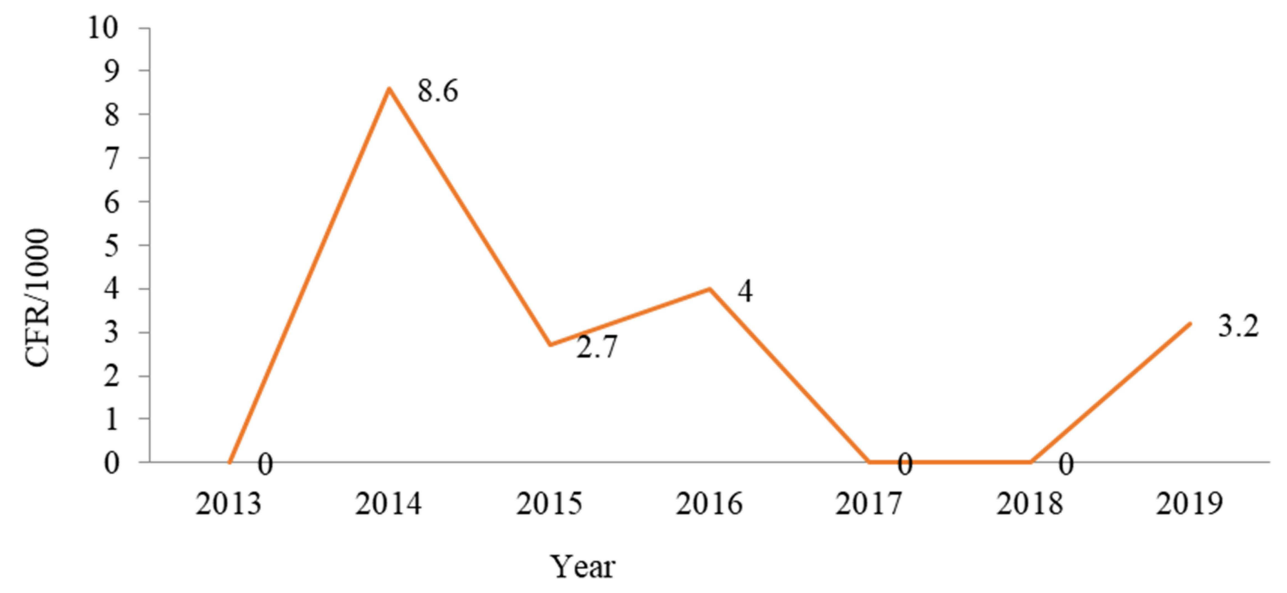

Figure 9 Trends in case fatality.

and their children miss vaccination both routine and supplementary immunization, which result in herd immunity.

Of the 993 serum samples, about 25\% were IgM-positive, $38 \%$ were discarded, and $14 \%$ were negative for measles IgM. This finding is in line with a study conducted in southern Ethiopia and Oromia region: $31.3 \%{ }^{18}$ $36 \%$ of samples IgM-positive, respectively. ${ }^{8}$ This is higher than confirmednational cases in 2008 and 2009: 10.7: and $9.1 \%$, respectively. ${ }^{16}$ This might be because measles is still prevalent and endemic in many districts of the zone. In other words, this finding indicates slight improvement in the strength of the case-based surveillance system of the zone.

A total of $13 \%$ of cases were admitted and $86 \%$ suspected cases treated as outpatients. The overall admission rate was $16 \%$, lower than a study conducted in Addis Ababa among 1,787 measles cases: 84\% were outpatients and the rest (16\%) inpatients. ${ }^{14}$ This might be due to limited resources among patients and shortages of admission rooms in health facilities in the study area.

The CSR was 3.07/1,000 population and high among children aged $<1$ year, followed by those aged 5-15 years. Fatality was higher among those of female sex than male: $3.3 / 1,000$ and 2.8/1,000, respectively. Fatality in the zone was low compared to a study in Niger, with a CRF of

Table 3 Vaccination status of measles cases by sex

\begin{tabular}{|l|l|l|l|l|}
\hline & Male & Female & $\mathbf{n}$ & $\%$ \\
\hline Vaccinated & 1,296 & 1,323 & 2,619 & 61.8 \\
Unvaccinated & 484 & 406 & 890 & 21 \\
Unknown & 369 & 363 & 732 & 17.3 \\
Total & 2,149 & 2,092 & 4,241 & 100 \\
\hline
\end{tabular}

9.7\% and highest CFR among infants (15.7\%), and those with nutritional deficiencies, eg, in vitamin A, showing higher fatality. ${ }^{19,20}$ The current expected CFR in Ethiopia is $3 \%-6 \%$; however, in certain high-risk populations, CFRs reach $30 \%$ in infants aged $<1$ year in the country. ${ }^{7}$ This is because the CFR for measles decreases with increasing age and is more fatal in those aged $<5$ years, due to poor nutritional and vitamin A supplementation.

Of the total cases, $62 \%$ were vaccinated. There were $21 \%$ unvaccinated patients, and $17 \%$ had unknown vaccination status. This is greater than a study conducted in Ethiopia, where $22 \%$ had received at least one vaccination, $24 \%$ were unvaccinated, and 54\% had unknown vaccination. $^{21}$ The study finding is lower than the study conducted in Oromia region, where 29\% were unvaccinated and $46 \%$ of with unknown vaccination status. ${ }^{8}$ It is also lower than the study conducted in Guji zone, where $77 \%$ were unvaccinated. ${ }^{18}$

Our analysis indicates that of all the cases reported, $27 \%$ were case-based reports and $73 \%$ by line lists, of which a majority $(72 \%)$ was reported in 2019 because of the outbreak. There were no reported cases by line list during 2018 or 2017. This shows there is sensitivity in the surveillance system and case notification is good. Casebased surveillance is part of the national PHEM system and a key component of the measles-control program. It is a system whereby every suspected measles case should be detected, reported, and undergo laboratory investigation. ${ }^{18}$

Regarding seasonal patterns, the most cases were reported during June (32\%), May (22\%), and July (12\%) and the least in October, November, and September, with $2 \%, 0.97 \%$, and $2.3 \%$, respectively. Cases reached their peak level in June, contrary to the findings of a study 


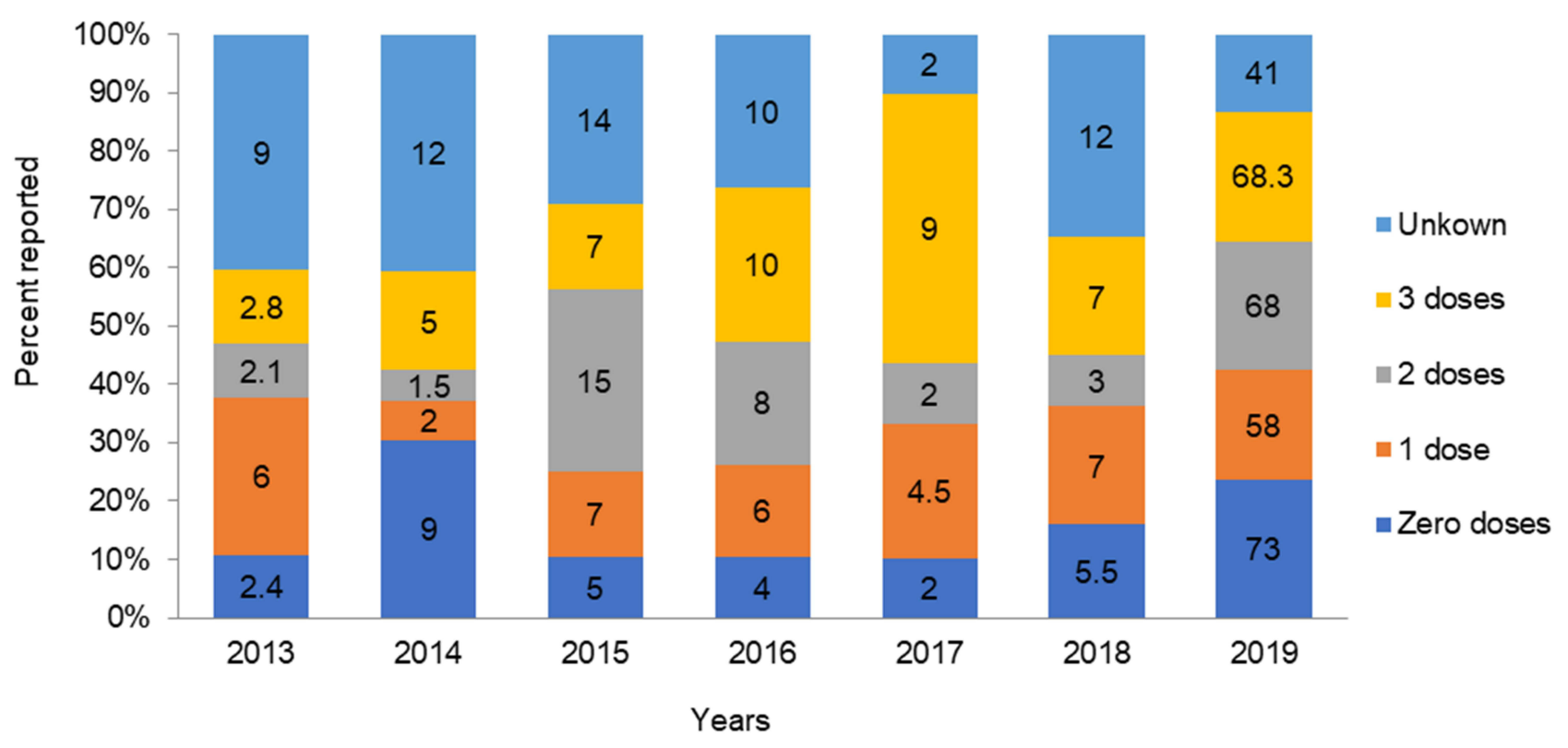

Figure 10 Vaccination status among reported cases.

conducted in Ethiopia, where there was a trend of increments in cases in January, February, and March. ${ }^{18,19}$ This is due to about $60 \%$ of the zone's climate being characterized by a long dry season.

The findings of this analysis revealed that only $21 \%$ of cases were reported by case-based investigation. WHO Africa recommends all measles cases be investigated using case-based investigation and samples be collected for all suspected cases. ${ }^{22}$ This study shows there are limitations in the implementation of case-based surveillance in the zone.

\section{Limitations}

We did not include analyses of laboratory samples collected by place or time, because the line-list and case-based reports were incomplete. Vitamin A-supplemented patients were not included, because deaths in the community were not reported and CFRs were underestimated. Time lines and completeness were not included because of incomplete data.

\section{Strengths}

This study tried to address all data types and variables. We included 2019 data because five districts of the zone were attacked by the outbreak. To analyze all related variables of the study, case-based reports and line lists were used.

\section{Conclusion}

Surveillance data on measles cases and geographic and age distribution can provide information about immunity gaps that are not identified by other monitoring methods alone. Generally, cases reported indicated a high CFRs compared to the expected 3\% in Ethiopia. The highest percentage of cases was seen in male patients. Despite the presence of effective vaccination, children aged $<4$ years were the most affected population. The highest numbers of cases were reported from Ginir and Dinsho districts, while Gura Damole and Gasera reported low numbers. Most of the samples examined showed IgM positivity, indicating high prevalence. Many in the study population were not vaccinated, which indicates a significant number of susceptible people in the community. The highest number was reported in 2019, contrary to the national measles-elimination strategy. Almost all suspected cases were treated as outpatients, with few cases being admitted. The cyclic pattern showed an increase in cases in April to June and reaching a peak in May.

\section{Recommendations}

Comprehensive measles surveillance needs to be enhanced to meet the elimination-strategy goal and surveillance-data collection and use for monitoring purposes of the elimination program improved. Health facilities and health offices of the districts in the zone have to investigate and report suspected cases and deaths in the community. Samples collected for laboratory confirmation should be improved in terms of quality and quantity by facilities. Routine immunization needs to be strengthened by health posts and health centers in the zone, in order to reduce the 
number of susceptible people. Even though different prevention strategies exist to expand immunization coverage, cases increased from 2013 to 2019. This raises questions about service quality, and health providers need to focus on quality improvement in immunization services. Support for the zone has to be strengthened in terms of early preparedness and supplementary immunization activities before April to overcome changes in seasonal patterns. Possible cause of measles in Ginir should be further investigated.

\section{Acknowledgments}

We would like to express our thank to God. We are grateful to the Ethiopian Field Epidemiology and Laboratory Training Program and Jimma University. We are also grateful to all Bale Zone Health Department staff for their cooperation in this study. Lastly, we would like to extend our gratitude to Bale field mentors and supervisors.

\section{Disclosure}

The authors report no conflicts of interest in this work.

\section{References}

1. Investment case for vaccine-preventable diseases surveillance in the African Region. 2020-2030. Brazzaville: WHO Regional Office for Africa; 2019. License: CC BY-CN-SA 3.0 IGO.

2. World Health Organization. Global vaccine-preventable diseases standards measles guide line. 2018:1-30.

3. Rewar S. Measles virus: a perpetual challenge Measles virus: a perpetual challenge. 2015.

4. WHO Regional Office for Europe. Guidelines for measles and rubella outbreak investigation and response in the WHO European Region: UN City, Marmovej 51, DK-2100 Copenhagen, Denmark, 2013.

5. Mohammed Y, Alemu AN. Measles outbreak investigation and response in Jarar Zone of Ethiopian Somali Regional State, Eastern Ethiopia. 2017.
6. 2015 AR measles and R progress update. Measles and rubella regional progress updates. 2015.

7. Ethiopian National measles guide line, 3rd Edition january 2012. Guideline on measles surveillance and outbreak management. 2012.

8. Akalu HB. Review on measles situation in Ethiopia; Past and Present. $J$ Trop Dis Public Health. 2015;4(2):2-7.

9. Orenstein WA, Hinman A, Nkowane B, Olive JM, Reingold A. Measles and rubella global strategic plan 2012-2020 midterm review. Vaccine 2018 Jan 11:36 Suppl 1:A34. doi:10.1016/j.vaccine.2017.09.026.pmid:29307367.

10. Asefa M. Addis Ababa University, College of Health Sciences, School of Public Health Addis Ababa University, College of Health Sciences, School of Public Health. 2018.

11. Goodson JL, Masresha BG, Wannemuehler K, et al. Changing epidemiology of measles in Africa. $J$ Infect Dis. 2011;204(Suppl 1): S205-S214. doi:10.1093/infdis/jir129

12. WHO Regional Office for Africa. African regional guidelines for measles and rubella surveillance. 2015.

13. Dabbagh A, Laws RL, Steulet C, et al. Progress toward regional measles elimination-worldwide, 2000 - 2017. Morb Mortal Wkly Rep. 2020;67(47):1323-1329. doi:10.15585/mmwr.mm6747a6

14. Hassen MN, Woyessa AB, Getahun M, et al. Epidemiology of measles in the metropolitan setting, Addis Ababa, Ethiopia, 2005 2014: a retrospective descriptive surveillance data analysis. $B M S$ Infect Dis. 2018;18:1-8.

15. European Centre for Disease Prevention and Control. Monthly measles and rubella monitoring report. 2018.

16. Belda K, Tegegne AA, Mersha AM, et al. Measles outbreak investigation in Guji zone of Oromia. Pan Afr Med J. 2017;27(Supp 2):1-5. doi:10.11604/pamj.supp.2017.27.2.10705

17. World Disasters Report 2016Resilience: saving lives today, investing for tomorrow.

18. Beyene BB, Ababa A, Tegegne AA, Enqueselassie F. National measles surveillance data analysis, 2005 to 2009, Ethiopia. 2016.

19. Nandy R, Handzel T, Zaneidou M, et al. Case-fatality rate during a measles outbreak in Eastern Niger in 2003. Clin Infect Dis. 2006;42:322-328. doi:10.1086/499240

20. Guidelines WHO, Preparedness E, Geneva MO. World Health Organization. 1999.

21. Federal Democratic Republic of Ethiopia Ministry of Health, Health Sector Transformation Plan I: AnualPerformance Report. 2019 EFY (2016/2017).

22. Thompson KM. What will it take to end fatalities from measles? Lancet Glob Heal. 2019;7(4):e394-e395. doi:10.1016/S2214-109X (19)30050-6

\section{Publish your work in this journal}

Risk Management and Healthcare Policy is an international, peerreviewed, open access journal focusing on all aspects of public health, policy, and preventative measures to promote good health and improve morbidity and mortality in the population. The journal welcomes submitted papers covering original research, basic science, clinical \& epidemiological studies, reviews and evaluations, guidelines, expert opinion and commentary, case reports and extended reports. The manuscript management system is completely online and includes a very quick and fair peer-review system, which is all easy to use. Visit http://www.dovepress.com/testimonials.php to read real quotes from published authors. 